\title{
Patient Handover as a Learning Activity for Medical Students
}

\author{
Michael L. Hoover \\ Educational and \\ Counselling Psychology \\ McGill University
}

\author{
Nancy Posel \\ Faculty of Medicine \\ McGill University
}

\author{
David Fleiszer \\ Department of Surgery \\ McGill University
}

\begin{abstract}
The move of medical education in North America to a model based on teaching competencies rather than knowledge requires new methods of assessment. As part of a longer-term project to create objective and quantifiable measures for the learning of these competencies, we argue that the focus should be on the Entrustable Professional Activities (EPAs) that are associated with each competency. In this paper we present a theoretical constructivist analysis of one such EPA, that of patient handover, which is critical to patient safety. By applying an Activity Theory analysis to this EPA, we were able to identify, and thus quantify, key components of the EPA. Analysis of student performance on an online virtual patient simulation of a trauma case indicated that surgical clerkship students were able to correctly mention the majority of the procedures that expert surgeons indicated were most important, but only used less than a third of the professional terms that experts thought appropriate. This result points to a need to increase direct instruction in professional communication among medical learners, while demonstrating that Activity Theory provides an analysis that not only captures what is happening in the learning, but also can be used to develop objective and quantifiable assessment metrics.
\end{abstract}

\section{Introduction}

Medical education in North America is in the process of moving from the century-old Flexner model towards a competency-based medical education (CBME) model, in which each required professional competency is associated with specified Entrustable Professional Activities (EPAs) ${ }^{[1][2][3]}$. This shift is being driven by the need for medical students to be ready to engage in professional activities on the first day of their residency. Both the Association of American Medical Colleges and the Association of Faculties of Education of Canada have specified core EPAs and their associated competencies and procedures. A central goal of CBME is to have a set of professional competencies whose associated EPAs can be objectively and quantifiably assessed. To address this question, we are engaged in a long-term project to create objective assessments for EPAs taught in Canadian medical schools. However, before there can be objective assessment of student performance on an EPA, there must first be an analysis of precisely what the EPA entails.

In this paper, we describe an analysis of teaching medical students one of the core competencies required in professional practice: that of effectively communicating information about a patient at the end of a shift or when handing over responsibility for a patient to a colleague, an authentic practice called patient handover. Failure to effectively and comprehensively communicate patient information at handover can severely compromise patient safety. Despite this, traditional medical instruction engaged in direct instruction of procedures for treating patients, trusting that students will simply pick up professional practices such as communication of the patient's conditions.

\section{Theoretical Framework}

Patient handover is a complex activity, involving several people, a deep understanding not only of the condition of the patient, but also of what information is most salient to the other health care professional, and how that information can be communicated most effectively and unambiguously. In order to capture the interaction of these elements in their complexity, we have used Engeström's ${ }^{[4]}$ Activity Theory (AT) framework as the basis for our analysis of patient handover.

AT is a modern instantiation of Vygotsky's social constructivist learning theory. Vygotsky[5] argued that human learning is not a passive process in which the learner simply receives the information that the instructor transmits. Instead learning consists of the learner building up a meaning that is shared by learners and instructors. Vygotsky described the process by which this happens in terms of the Zone of Proximal Development in which learners are guided by the instructor or advanced peers through an activity that the learners can complete with support, but cannot yet perform on their own. Engeström, building on work by Leont' ${ }^{[6]}{ }^{[6]}$, fleshed out this process and identified the core components of such learning activities.

AT has been used successfully in health contexts to describe and understand complex medical phenomena. Several studies have used AT to 
describe the nature of the relationship between members of the medical community ${ }^{[7][8][9]}$, indicating that a tendency towards hierarchical relationships in hospitals impedes successful achievement of a shared objective. Foot ${ }^{[10][11]}$ used AT to analyze the practices of Norwegian social service professionals, with important implications for practice. In a recent study, Almalki, Gray and Martin-Sanchez ${ }^{[12]}$ used an AT analysis of published articles on health selfquantification to argue that although across articles the full range of the activity was studied, no single article addressed the activity as a whole. In studies of patient behavior, Koski-Jänne' ${ }^{[13]}$ AT analysis of addictive behaviour demonstrated that the addict engages in non-optimal actions that result in contradictions that can impede successful recovery, while Hoover and Carani ${ }^{[14]}$ used AT to analyze the mental schemas that distinguished medically compliant and non-compliant people living with AIDS.

Recently, several papers ${ }^{[15][16]}$ have argued that AT should be used as a research framework for studying medical education because it can capture the complexity of medical practice. Beyond their arguments, we propose that the growing emphasis on using EPAs as milestones in medical education makes the AT approach even more appropriate because the AT framework allows us to look at the EPA as an activity to be learned, rather than as a series of pieces of information which must somehow be brought together at the right time.

\section{A Brief Overview of Activity Theory}

Activity Theory (AT) differs from cognitive learning theories in five important ways, all drawn from the social constructivist learning theory of Vygotsky. The first is that human learning is not seen as the transfer of information to learners, nor the passive absorption of information, but rather as the learners actively constructing their mental representations. Second, this construction of meaning is the result of engagement with others on a task with a specific objective: that is, effective learning only occurs when learners engage with a support community of more knowledgeable instructors or peers, who guide them through a task that they are able to do with support, but not yet without it. The result of repeated social engagement on a task is the building up, or construction, of a mental schema for the activity. Thus any learning activity has three central components: the learner, the learning objective, and the community supporting the learning.

The third core idea in AT also comes from Vygotsky: that which distinguishes human from animal learning is the use of mediators. Rather than engaging directly with the learning objective, human learners employ a set of tools, which can be concrete or psychological. For example, in a lesson about addition, a teacher might use concrete tools such as chalk and a blackboard, but will also use psychological tools such as the language (e.g., English), symbols (such as the plus or equals sign) as well as words that have a specific meaning in that context (such as the word 'plus', which has other meanings in other contexts, but is unambiguous in maths). Each pair of the central components has its own set of mediators: the relation between the learner and the object is mediated by concrete and psychological tools, the interaction of the learner and the support community is mediated by the rules for communication (such as turn-taking), and the relation between the support community and the learning objective is mediated by the different roles that community members can take on (such as teacher as coach or model.) The relationship of the three central components plus the three kinds of mediators can be represented using Engeström's activity triangle (see Figure 1).

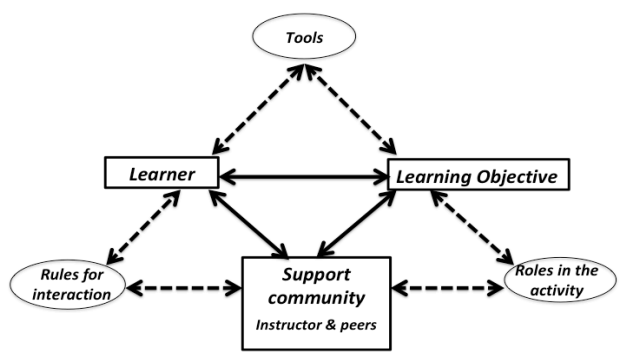

Figure 1: A representation of a learning activity with mediators in circles. (after Engeström ${ }^{[17]}$ )

Fourth, this approach requires that the unit of analysis be the activity as a whole, and not any cognitive subcomponents that might be employed in the activity (see Davidoff ${ }^{[18]}$ for a brief discussion). This is because components and mediators interact with each other in unique ways in a given activity.

Fifth, and finally, the AT approach recognizes that learners and the community, and therefore the learning, takes place with a specific cultural context. While 'learner', 'community' and 'objective' are universal across all learning environments, the mediators, 'tools', 'rules' and 'roles', are determined by the specific cultural context. In the case of medical students, they are enmeshed in a specific cultural context (for example that of an Englishspeaking institution in Canada) that dictates that (e.g.) English will be the language of learnercommunity interaction, and that the norms for interaction will be those of the general culture. Beyond this, the learning is also taking place within a specific subcultural setting: that of an English Canadian teaching hospital. As such, the roles that 
the different actors can take on are affected by the hierarchical nature of the institution, the rules for interaction between members follow the norms of the profession and the institution, and the psychological tools that are used are specific to medicine. Just as the word 'plus' in a maths class unambiguously refers to addition, there are terms used in hospital that have specific, unambiguous meanings in that context, such as 'abduction', or 'benign', as well as words that are used to indicate a specific medical condition, such as 'primapara' or 'mastitis'.

\section{Activity Theory Analysis of the Patient Handover}

The EPA of professional communication can be represented as a key professional activity, that of patient handover: the communication of the important elements of a patient's case to a colleague to whom responsibility for the case is being transferred. We can analyse this activity as consisting of the following three central components:

The Objective: production of a handover, a clear and comprehensive description of the patient's condition and treatment needs.

The Learner: the medical student engaging in a clerkship in surgery.

The support Community: the fellow students, the residents, and the professor of surgery.

These are then mediated by the:

Rules for interaction: when and how the medical student provides the handover summary to the other members of the community. How and to whom the learner can ask questions, and ask for help.

Roles: the medical student is being trained, through the activity, to take on the role of physician (in training), providing needed information to other hospital staff members. The instructor meanwhile takes on the role of expert, providing a model of a comprehensive handover, and feedback on the student's handover.

Psychological Tools: the mental schema or schemas that the medical student must possess to engage in the activity of handover. Before students can successfully engage in the activity, they must already have these schemas - learning to engage in the activity thus becomes an exercise in applying schemas that they have already learned.

Before students can successfully engage in a learning activity such as creating a patient handover, they must have already mastered the tools, rules and roles needed for the activity. If any of these are absent from the student's repertoire, then the learning activity will fail. Remediation consists of identifying the missing mediator, and including its teaching as a precursor to the learning activity.
Although the rules of interaction and the roles played are vitally important for effective learning, in the present paper we will concentrate on the tools, and especially the psychological tools that are needed to engage in the activity under study.

In order to produce objective assessments for students' handovers, we employed a Virtual Patient Simulation (VPS) in which medical students were presented a trauma case online, with photos and test reports, and were asked to decide which procedures, including further tests, should be done, and then to communicate that information as they would to a colleague who was taking over the case. The advantages of using a VPS are several: it standardizes the cases across a large number of students, it allows the controlled presentation of all needed information, and it permits immediate comparison of student handovers to those of experts who have engaged in the VPS earlier. More importantly, it permits the use of the experts' handover as a template for objectively assessing students' handovers. Elsewhere ${ }^{[19]}$, we have described the development of such assessment tools based on a VPS, in which we identified the key terms that experts included in their handover summaries, and used these to create assessment rubrics. These rubrics were used by non-expert raters to assign a score to each student's handover based on whether the student included each item in their handover for the VPS. In this paper, we focus on the theoretical analysis that underlies the creation of not one but two assessment tools for the same VPS.

Analysis of the students' handovers after they had completed their surgical clerkship revealed that, while they made reference to $61 \%$ of the medical procedures that experts mentioned, they used only $31 \%$ of the appropriate semantic medical terms that would effectively and unambiguously communicate the patient's condition to colleagues.

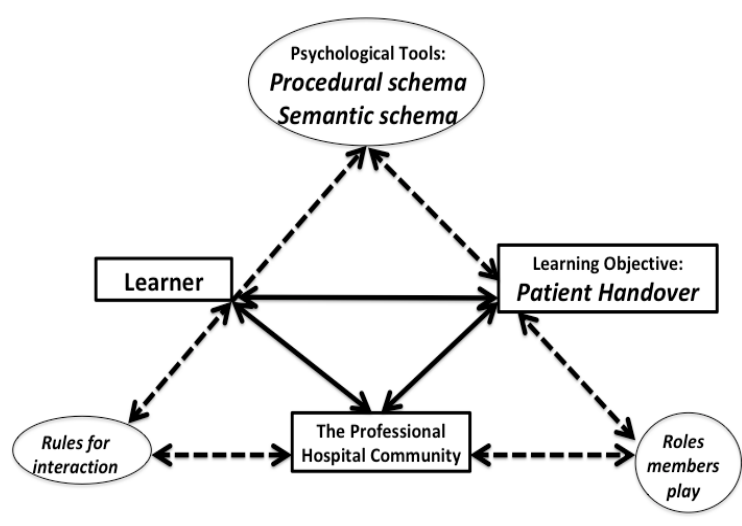

Figure 2. Activity Theory analysis of patient handover as an activity

This analysis indicates that there were two psychological tools (or mental schemas) needed to 
engage in the activity of handover: an understanding of the procedures that are needed (a procedural schema), and a mastery of the precise medical terms used when speaking about patients and their care (a semantic schema). Figure 2 presents these schemas as mediators between the learner and the objective of patient handover.

\section{Discussion}

In describing the psychological tools that a medical student needed to produce an effective handover, we were able to objectively distinguish two kinds of knowledge that are needed to engage in the EPA of patient handover: the knowledge of the medical procedures required by a patient's condition, and the knowledge of the semantic terms needed to describe that patient's condition precisely and unambiguously to colleagues. That is, medical students not only need to learn what procedures are needed in (e.g.) a trauma case, they must also learn how to talk about the case using words and phrases whose meaning is both unambiguous and shared by other members of the medical community. Crucially, patient handovers occur within a specific professional setting that - consistent with Vygotsky's construct of 'word meaning' - requires the use of specific words and terms (semantics) that convey specific meanings within that setting. In trauma cases, certain words/terms have meanings that are extremely specific and convey information accurately once people share those meanings. To put it another way, the use of medical 'jargon' in a hospital setting does not have its roots in an attempt to mark the in-group, nor to hide information from patients and family, but rather serves to reduce ambiguity and increase clarity. Medical students' inability to use the expected medical terminology in a patient handover can lead to misunderstandings which can have serious negative effects on patient outcome.

\section{Conclusions}

This analysis of medical students learning the required EPA of patient handover, lends strong support for the arguments put forth by Frambach, Driessen, and van der Vleuten ${ }^{[15]}$, and Johnston and $\operatorname{Dornan}^{[16]}$ for the use of activity theory as a framework for studying medical education. Through its use, we were able to identify and describe the different kinds of knowledge that students need in order to carry out this professional activity. Although the AT approach has been traditionally used as a qualitative methodology, our analysis shows that by using expert-based rubrics, it can also be used to develop effective quantitative analysis.

More importantly, this analysis points to an area where training in this EPA needs to be improved.
The traditional training was effective in teaching medical students to identify the appropriate medical procedures, but less so in using the precise and unambiguous language that experts use to effectively communicate to colleagues. This points to a need to provide direct training of the semantic schema needed to engage in the EPA of professional communication.

\section{References}

[1] Carraccio, C., Wolfsthal, S.D., Englander, R., Ferentz, K., and C. Martin, "Shifting paradigms: from Flexner to competencies", Academic Medicine, 2002, pp. 361-367.

[2] Association of American Medical Colleges, "Core entrustable professional activities for entering residency (CEPAER)", AAMC Spring Meeting Update 2014. https://www.aamc.org/download/379308/data/coreentrusta bleprofessionalactivities.pdf.

[3] Association of American Medical Colleges. "Core entrustable professional activities for entering residency: Curriculum developers' guide", 2014. https://members.aamc.org/eweb/upload/Core\%20EPA\%20 Curriculum\%20Dev\%20Guide.pdf

[4] Engestrom, Y. Expansive learning at work: toward an activity theoretical reconceptualization. Journal of Education and Work, 2001, pp. 133-156.

[5] Vygotsky, L. Mind in society: The development of higher psychological processes, Cambridge, MA, Harvard University Press, 1978.

[6] Leont'ev, A. N., "The Problem of Activity in Psychology”, Soviet Psychology, 1974, pp. 4-33.

[7] Allard, J. and A. Bleakley, "What would you ideally do if there were no targets? An ethnographic study of the unintended consequences of top-down governance in two clinical settings", Advances in Health Sciences Education, 2016, pp. 803-817.

[8] Kerosuo, H., and Y. Engeström, "Boundary crossing and learning in creation of new work practice", Journal of Workplace Learning, 2013, pp. 345-351.

[9] Edwards, A., "Working Collaboratively to Build Resilience: A CHAT Approach", Social Policy and Society, 2007, pp. 255-264.

[10] Foot, K., "Cultural-Historical Activity Theory: Exploring a Theory to Inform Practice and Research", Journal of Human Behavior in the Social Environment, 2014, pp. 329-347.

[11] Foot, K., "Using cultural-historical activity theory to analyze social service practices evolving from the Norwegian HUSK projects", Journal of EvidenceInformed Social Work. 2015, pp. 112-123.

[12] Almalki, M., Gray, K., and F. Martín-Sanchez, "Activity Theory as a Theoretical Framework for Health 
Self-Quantification: A Systematic Review of Empirical Studies", Journal of Medical Internet Research, 2016, pp. e131.

[13] Koski-Jännes, A., "From addiction to selfgovernance", pp. 435-444 in Y. Engeström, R. Miettinen and R-L. Punamäki (Eds.) Perspectives on Activity Theory. Cambridge: Cambridge University Press. 1999.

[14] Hoover, M. L., and G. Carani, "Medical Adherence as an Activity." Paper presented at the triennial meeting of the International Society for Cultural-Historical Activity Research (ISCAR), Quebec City, 2017.

[15] Frambach, J. M., Driessen, E. W., and C. P. M. van der Vleuten, "Using activity theory to study cultural complexity in medical education." Perspectives on Medical Education, 2014, pp. 190-203.

[16] Johnston, J., and T. Dornan, "Activity theory: Mediating research in medical education”, pp. 99-104 in J. Cleland and S. J. During (Eds.), Researching Medical Education, Chichester: Wiley Blackwell, 2015.

[17] Engeström, Y., Learning by Expanding. Cambridge: Cambridge University Press, 2014.

[18] Davidoff, V.V. "The Content and unsolved problems of Activity Theory", pp. 29-52 in Y. Engeström, R. Miettinen and R-L. Punamäki (Eds.) Perspectives on Activity Theory, Cambridge, Cambridge University Press, 1999.

[19] Fleiszer, D., Hoover, M. L., Posel, N., and S. Bergman, "Development and validation of a tool to evaluate clinical reasoning in trauma using virtual patients", Journal of Surgical Education. 2018, pp. 779786. 